\title{
INFLUENCE OF SURGICAL TECHNIQUE ON PAIN AND POSTOPERATIVE STAY OF PATIENTS SUBJECTED TO AMBULATORY HEMORRHOIDECTOMY
}

GIL BONA J., MARTÍNEZ UBIETO F., GRACIA ROCHE C., PEÑA CALVO P., MARTÍNEZ UBIETO J., PASCUAL BELLOSTA A. DEPARTMENT OF ANESTHESIOLOGY. MIGUEL SERVET HOSPITAL. ZARAGOZA.

\section{OBJECTIVE}

To assess the influence of postoperative pain on the postoperative stay of patients undergoing ambulatory hemorrhoidectomy, and its incidence depending the type of surgical technique performed.

\section{MATERIAL AND METHOD}

We performed a prospective study over patients undergoing ambulatory major surgery from June 2013 to June 2016. 120 patients Undergoing hemorrhoidectomy (58 subjected to a Hemorrhoidal Laser Technique, HeLP, and 62 subjected to a Milligan-Morgan technique, MM) were introduced.

All patients were subjected to a balanced general anesthesia using a laryngeal mask.

We made a comparative analysis evaluating pre, intra, and post-operative data according patients were operated under a HeLP technique or a MM technique.

To correlate different variables we used the chi square of Pearson or Fisher test for discrete variables and t-student or $U$ Mann-Whitney test for continuous variables. Significance at $\mathrm{p}<0.05$

\section{RESULTS}

\begin{tabular}{|c|c|c|c|}
\hline DATA & HeLP technique & MM technique & $\mathbf{P}=$ \\
\hline Number & 58 & 62 & \\
\hline PREOPERATIVE & & & \\
\hline$\%$ Mens & $51.7 \%$ & $56.5 \%$ & 0.603 \\
\hline Age & $50.7+/-12.8$ & $50.7+/-12.4$ & 0.165 \\
\hline$A S A=1$ Vs $A S A=2$ & $60,3 \%$ Vs $39.7 \%$ & $59.7 \%$ Vs $40.3 \%$ & 0.941 \\
\hline Hypertension & $20.7 \%$ & $35.5 \%$ & 0.072 \\
\hline Hyperlipemia & $19 \%$ & $27.4 \%$ & 0.274 \\
\hline Obesity & $6.9 \%$ & $16.1 \%$ & 0.115 \\
\hline INTRAOPERATIVE & & & \\
\hline Hemorroidal grade (III Vs IV) & $89.7 \%$ Vs $10.3 \%$ & $80.6 \%$ Vs $19.4 \%$ & 0.167 \\
\hline Intraoperative complications & $5.1 \%$ & $6.4 \%$ & 0.929 \\
\hline Surgical time (minutes) & $46.69+/-5.28$ & $37.31+/-9.4$ & $<0.001$ \\
\hline POSTOPERATIVE & 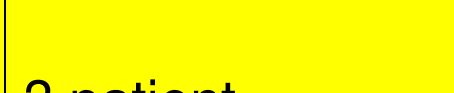 & 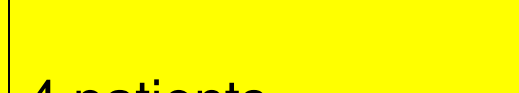 & 0619 \\
\hline $\begin{array}{l}\text { HOSPITAL INCOME NEED } \\
\text { Recoverv time(min) }\end{array}$ & $\begin{array}{l}3 \text { patient } \\
1319+/-1553\end{array}$ & $\begin{array}{l}4 \text { patients } \\
23266+/-3426\end{array}$ & $<0.001$ \\
\hline $\begin{array}{l}\text { Recovery time (min) } \\
\text { Urinate delay }\end{array}$ & 2 patients $(3.4 \%)$ & 1 patient $(1.6 \%)$ & 0.941 \\
\hline Oral intolerance & 1 patient $(1.7 \%)$ & 1 patient $(1.6 \%)$ & $>0.9$ \\
\hline Difficult control of pain & 0 patients $(0 \%)$ & 11 patients $(17.7 \%)$ & $<0.001^{\prime}$ \\
\hline VAS $12 \mathrm{H}$ after surgery $>3$ & $5.2 \%$ & $62.9 \%$ & $<0.001$ \\
\hline VAS $48 \mathrm{H}$ after surgery>3 & $13.8 \%$ & $79 \%$ & $<0.001^{\prime}$ \\
\hline VAS 7 days after surgery>3 & $0 \%$ & $54.8 \%$ & $<0.001^{\prime}$ \\
\hline NSAIDs doses $12 \mathrm{H}$ after surg. & 2.67 & 4.31 & $<0.001$ \\
\hline NSAIDs doses $48 \mathrm{H}$ after surg. & 2.78 & 4.37 & $<0.001$ \\
\hline NSAIDs doses 7 days after surg. & 0.55 & 3.19 & $<0.001^{\prime}$ \\
\hline
\end{tabular}

\section{CONCLUSIONS}

Post-operative pain was the main cause of late discharge in patients undergoing ambulatory hemorrhoidectomy

Pain and analgesic requirements in the first 7 postoperative days were significantly lower when hemorrhoidectomy was performed using HeLP technique compared to MM technique 